The Astrophysical Journal Supplement Series, 90:639-643, 1994 February

(C) 1994. The American Astronomical Society. All rights reserved. Printed in U.S.A.

\title{
GAMMA-RAY SPECTRAL OBSERVATIONS WITH YOHKOH
}

\author{
M. Yoshimori, K. Suga, K. Morimoto, T. Hiraoka, and J. Sato \\ Department of Physics, Rikkyo University, Toshima-ku, Tokyo 171, Japan \\ K. KaWABATA \\ Department of Physics and Astrophysics, Nagoya University, Nagoya 464-01, Japan \\ AND \\ K. OHKI \\ National Astronomical Observatory, Mitaka, Tokyo 181, Japan \\ Received 1993 March 1; accepted 1993 May 19
}

\begin{abstract}
We present gamma-ray observations of the 1991 October 27, November 15, and December 3 flares with the Yohkoh gamma-ray and hard X-ray spectrometers. The October 27 flare (X6.1/3B) shows significant gamma-ray line emission, and the temporal evolution of the gamma-ray line-to-bremsstrahlung flux ratio indicates that protons and electrons were complicatedly accelerated during flare. The December 3 flare (X2.0/2B) shows a strong bremsstrahlung continuum extending to $10 \mathrm{MeV}$ and indicates that electrons were preferentially accelerated to $10 \mathrm{MeV}$. A line feature at $420 \mathrm{keV}$ was observed from the November 15 flare (X1.0/3B). This is most likely due to a compound of redshifted nuclear deexcitation lines of $\mathrm{Be}(429 \mathrm{keV})$ and $\mathrm{Li}(478 \mathrm{keV})$ resulting from $\mathrm{He}-\mathrm{He}$ reactions. The spectral feature indicates that the accelerated He nuclei suffer strong pitch-angle scattering in the corona and form a downward-peaked distribution. Furthermore, the November 15 flare exhibits evidence of positron annihilation line at $511 \mathrm{keV}$. The positron production processes and the electron density of the annihilation region are discussed on the basis of the temporal characteristics of $511 \mathrm{keV}$ line emission.
\end{abstract}

Subject headings: line: identification — Sun: flares — Sun: X-rays, gamma-rays

\section{INTRODUCTION}

The gamma-ray spectrum is composed of two components, electron bremsstrahlung continuum and characteristic lines of nuclear origin. The time profiles and energy spectra for both components provide essential diagnostics for electron and proton energization processes during solar flares. SMM (Chupp 1987; Rieger 1989) and Hinotori (Yoshimori 1989) observed a large number of gamma-ray flares in the last solar maximum, and the particle acceleration and gamma-ray production processes have been widely studied. However, the particle acceleration mechanism, which is the most important for the understanding of high-energy solar flare phenomena, has not yet been well solved so far.

Yohkoh was launched in 1991 August to observe high-energy solar flare phenomena with imaging and spectroscopic instruments. The gamma-ray spectrometer consists of two identical bismuth germanate (BGO) scintillation detectors ( 3 inches in diameter and 2 inches in thickness) and covers the $0.4-100 \mathrm{MeV}$ band. It has an improved detection sensitivity to gamma-rays in the $1-10 \mathrm{MeV}$ band. The hard X-ray spectrometer consists of a $\mathrm{NaI}$ ( T1 ) scintillation detector ( 3 inches in diameter and 1 inches in thickness) and covers the $20-600 \mathrm{keV}$ band. Detailed descriptions of the instrument can be found elsewhere (Yoshimori et al. 1991).

The gamma-ray spectrometer recorded several X-class flares with photon emission above $1 \mathrm{MeV}$ between 1991 October and December. In the present paper, we discuss the following problems on the basis of the time profiles and energy spectra of gamma-ray flares observed with Yohkoh: (1) timings of electron and proton acceleration, $(2)$ proton energy spectra in the
10-200 MeV band, (3) angular distribution of accelerated $\mathrm{He}$ nuclei, and (4) production of the positron annhilation line at $511 \mathrm{keV}$.

\section{OBSERVATIONS}

Most gamma-ray flares have been known to emit strong nuclear lines in the 1-7 MeV band. A typical gamma-ray line flare (X6.1/3B, location S13 E15) was observed in 05:4005:42 UT on 1991 October 27 (Yoshimori et al. 1992a, b). The flare lasted until 0544 UT (G. Trottet, private communication), but the flare observation was terminated due to switching of the observation mode before the flare was over. The time profiles in the 0.4-0.7 and 4-7 MeV bands are shown in Figure 1. The time resolution is $4 \mathrm{~s}$. The temporal variations are of impulsive characteristics in gamma-ray energies. A spectral deconvolution procedure was applied to derive a photon spectrum from the energy-loss distribution. The photon spectrum, integrated over the maximum phase of the flare $05: 40: 31-$ $05: 41: 15$ UT), is shown in Figure 2. The spectrum is dominated by nuclear lines above $1 \mathrm{MeV}$, and shows nuclear deexcitation lines of $\mathrm{Fe}(1.25 \mathrm{MeV}), \mathrm{Mg}(1.37 \mathrm{MeV}), \mathrm{Ne}(1.63$ $\mathrm{MeV}), \mathrm{Si}(1.78 \mathrm{MeV}), \mathrm{O}+\mathrm{Ne}+\mathrm{Mg}$ lines around $2.7 \mathrm{MeV}, \mathrm{C}$ $(4.44 \mathrm{MeV})$, and $\mathrm{O}(6.13 \mathrm{MeV})$ and neutron-capture line at 2.22 MeV. As shown in Figure 1, the time profiles of the electron bremsstrahlung radiation $(0.4-0.7 \mathrm{MeV})$ and nuclear lines (4-7 MeV) do not exhibit close similarities.

Another type of gamma-ray flare (X2.0/2B, location N17 E72) was observed in 16:36-16:37 UT on 1991 December 3 (Yoshimori et al. 1992a). The flare shows a strong power-law spectrum extending to $10 \mathrm{MeV}$. The photon flux spectrum, 

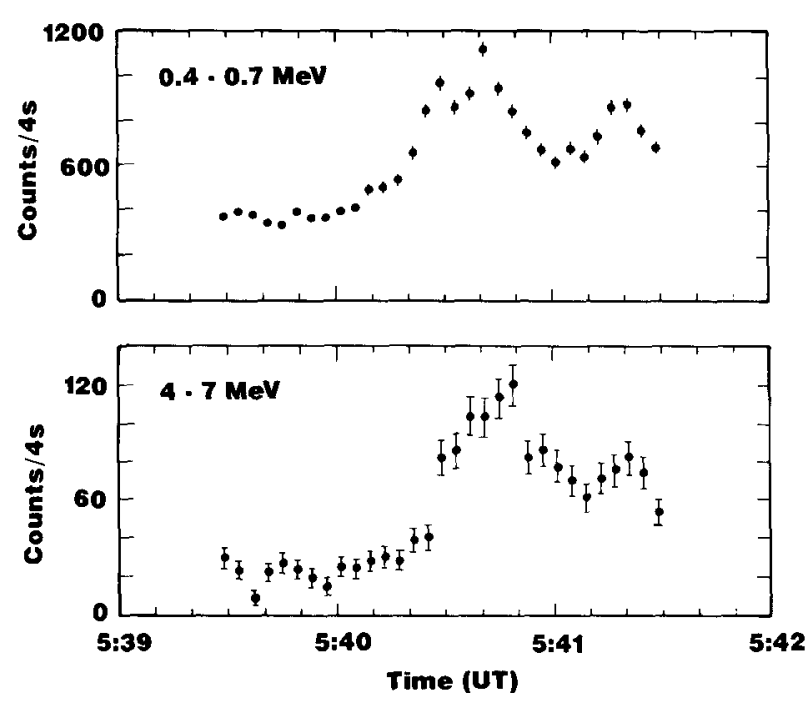

Fig. 1.-Time profiles of the $0.4-0.7$ and $4-7 \mathrm{MeV}$ bands for the $199 \mathrm{I}$ October 27 flare.

integrated over the maximum phase of the flare (16:36:2016:36:28 UT), is shown in Figure 3. It is dominated by electron bremsstrahlung over the entire energy range, and the contribution of nuclear lines is not significant.

A line feature at $420 \mathrm{keV}$ was observed from the 1991 November 15 flare $(\mathrm{X} 1.0 / 3 \mathrm{~B}$, location $\mathrm{S} 12 \mathrm{~W} 13)$ with the hard $\mathrm{X}$-ray spectrometer. The line feature was detected at the peak time of the flare $(22: 37: 48-22: 37: 52$ UT). The photon spectrum in the $20-600 \mathrm{keV}$ band, integrated for $4 \mathrm{~s}$ at the peak time, is shown in Figure 4. At the peak time, a counting loss occurred due to the high counting rate. Small corrections for a dead time was made to derive the photon spectrum. The line feature is most likely due to a compound of the Dopplershifted 429 and $478 \mathrm{keV}$ lines due to excited Be and Li nuclei produced by interactions of accelerated He nuclei with ambient He in the solar atmosphere (Kozlovsky \& Ramaty 1974, $1977)$. The fluence in the line feature is $(4.2 \pm 0.8)$ photons $\mathrm{cm}^{-2}$.

Furthermore, evidence for the positron annihilation line at $511 \mathrm{keV}$ was observed from the November 15 flare with the gamma-ray spectrometer. The line was detected during the decay phase $(22: 37: 50-22: 38: 26 \mathrm{UT})$. The count spectrum in the $400-800 \mathrm{keV}$ band, integrated over 22:37:50-22:38:26 $\mathrm{UT}$, is shown in Figure 5. The derived fluence in the $511 \mathrm{keV}$ line was $(3.2 \pm 1.0)$ photons $\mathrm{cm}^{-2}$. However, the $511 \mathrm{keV}$ line was not apparent at the peak time (22:37:48-22:37:50 UT), as shown in Figure 4, because it has delayed characteristics.

\section{DISCUSSION}

The October 27 flare shows significant bremsstrahlung emission below $1 \mathrm{MeV}$ and gamma-ray lines in the $1-7 \mathrm{MeV}$ band. Most gamma-ray flares observed with SMM and Hinotori in the last solar maximum exhibited a similar spectral feature. This implies that protons and ions were efficiently accelerated to high energies above $10 \mathrm{MeV}^{\text {nucleon }}{ }^{-1}$ in a short time scale. The temporal evolution of the ratio of the counting rate in the 4-7 MeV band (gamma-ray lines are dominant) to the counting rate in the $0.4-0.7 \mathrm{MeV}$ band (bremsstrahlung is dominant) is shown in Figure 6. It gives an important key to study the timings of electron and proton acceleration during the flare. Figure 6 shows that the ratio is variable with time. It indicates that the electrons and protons were not simultaneously accelerated or the efficiency of proton acceleration

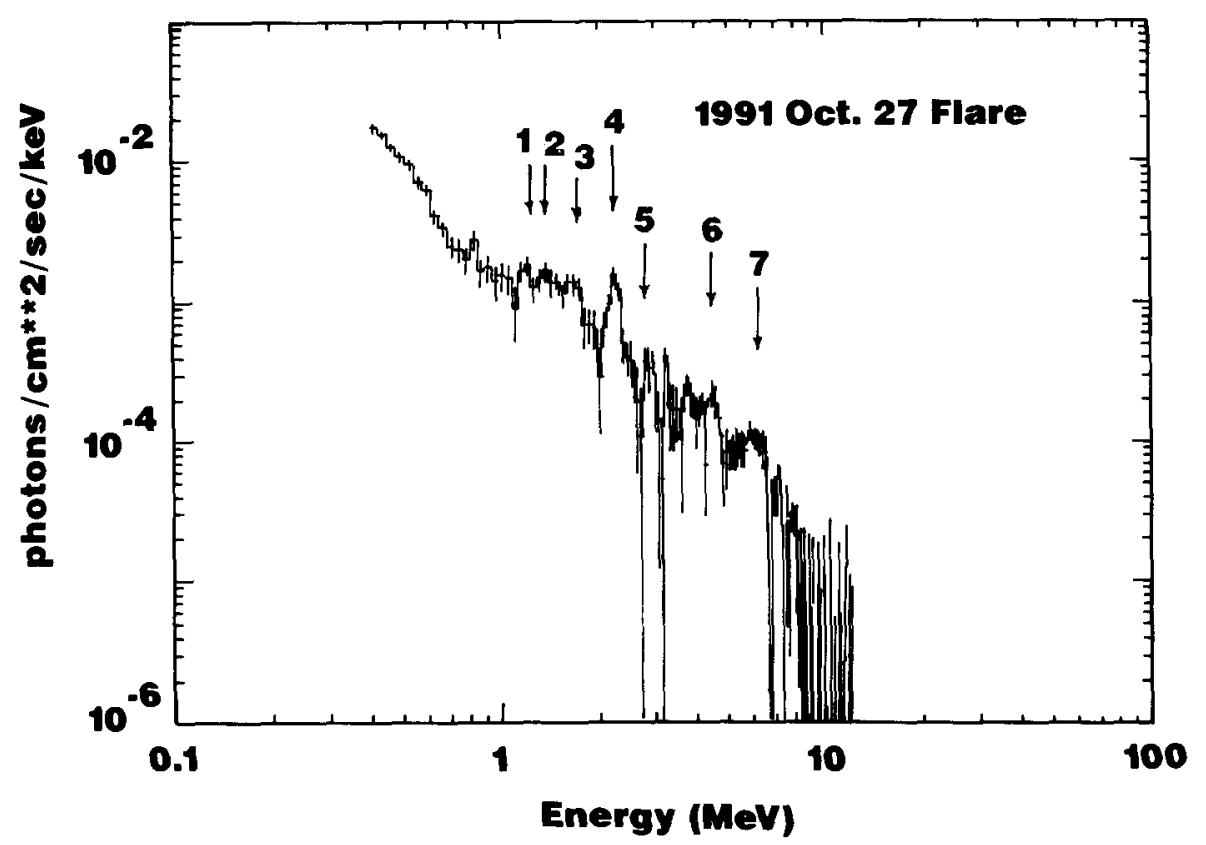

FIG. 2.-Gamma-ray spectrum for the 1991 October 27 flare $(05: 40: 31-05: 41: 15 \mathrm{UT}$ ). Seven arrows correspond to gamma-ray line energies ( 1 : Fe line at $1.25 \mathrm{MeV} ; 2: \mathrm{Mg}$ line at $1.37 \mathrm{MeV} ; 3: \mathrm{Ne}$ line at $1.63 \mathrm{MeV}+$ Si line at $1.78 \mathrm{MeV} ; 4$ : neutron capture line at $2.22 \mathrm{MeV} ; 5: \mathrm{O}+\mathrm{Ne}+\mathrm{Mg}$ lines around 2.7 $\mathrm{MeV} ; 6: \mathrm{C}$ line at $4.44 \mathrm{MeV} ; 7: \mathrm{O}$ line at $6.13 \mathrm{MeV}$ ). 


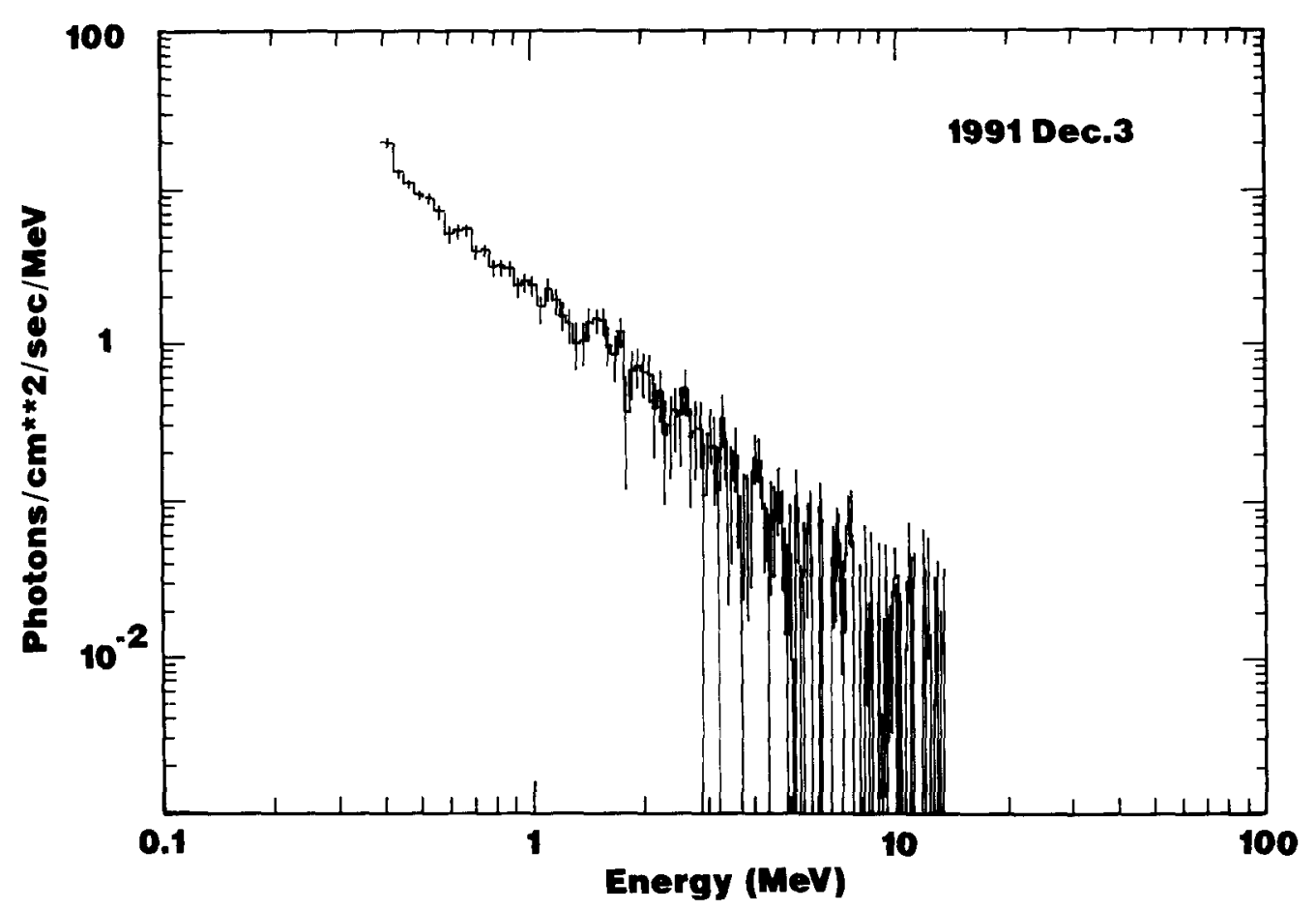

FIG. 3.-Gamma-ray spectrum for the 1991 December 3 flare (16:35:20-16:35:28 UT)

(a)
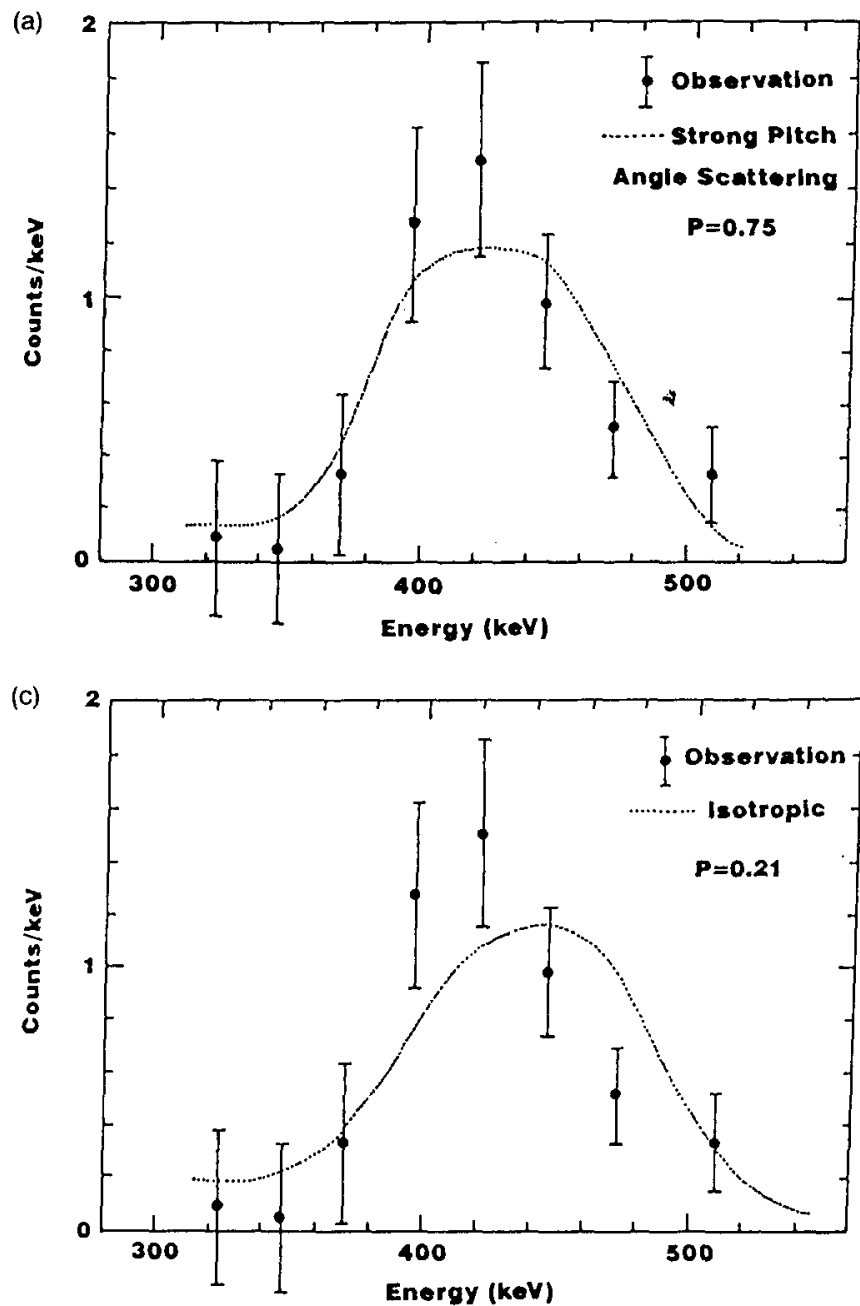

(b)

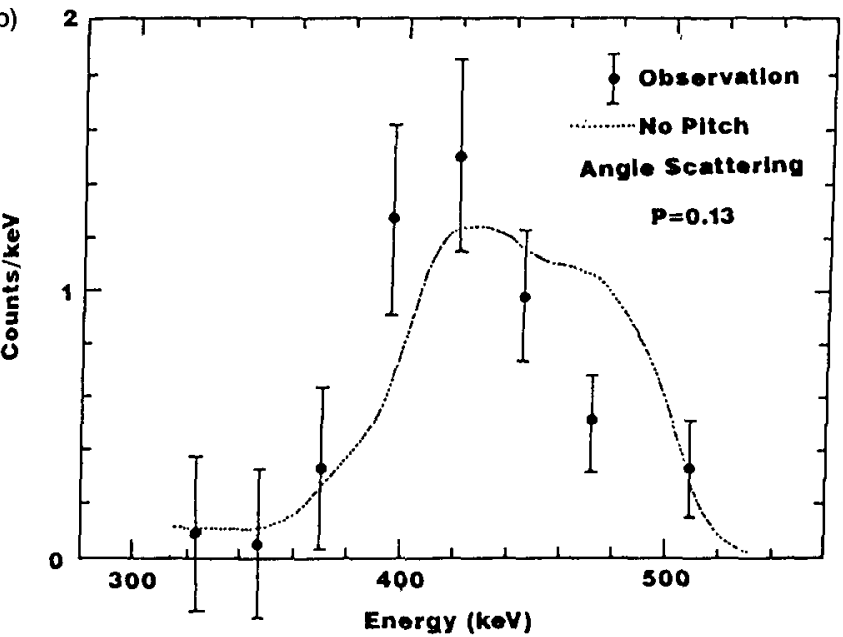

FIG. 4.-Continuum-subtracted spectrum of the Li-Be lines for the 1991 November 15 flare. Calculated spectra for three models $(a)$ strong pitch-angle scattering, $(b)$ no pitch-angle scattering, and $(c)$ isotropic distribution) are shown for comparison. 


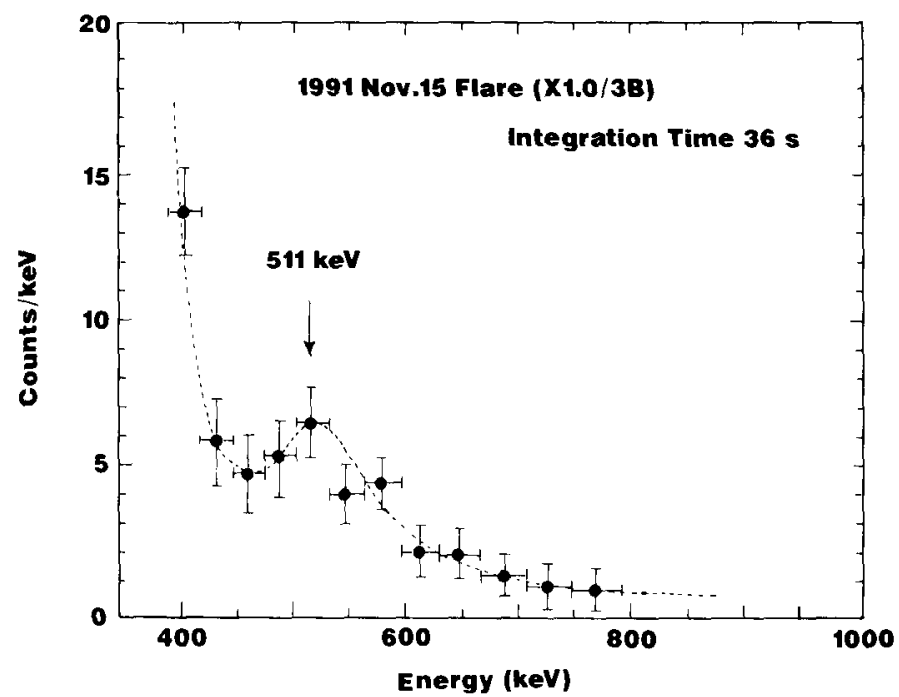

FiG. 5.-Count spectrum of the $511 \mathrm{keV}$ line for the 1991 November 15 flare.

increases with time relative to electron acceleration. It means that both particles were complicatedly accelerated during the flare.

On the other hand, the December 3 flare showed a strong bremsstrahlung continuum extending to $10 \mathrm{MeV}$, but did not show significant gamma-ray line emission. This implies that electrons were preferentially accelerated to high energies during the flare. This type of spectrum has been observed from a small number of gamma-ray flares (Rieger \& Marschhäuser 1990) and is called an electron-dominated event. The ratio of the counting rate in the $4-7 \mathrm{MeV}$ band to the counting rate in the $0.4-0.7 \mathrm{MeV}$ band is shown in Figure 7. The ratio is constant within the statistical errors during the flare. It implies that electrons were simultaneously accelerated to $10 \mathrm{MeV}$ with the low-energy electrons.

A proton energy spectrum can be derived from the fluence ratio of the nuclear deexcitation lines to the neutron capture line at 2.22 MeV (Murphy \& Ramaty 1984; Yoshimori \& Watanabe 1985; Hua \& Lingenfelter 1987). The ratio is most sensitive to the proton spectrum in the $10-100 \mathrm{MeV}$ range.

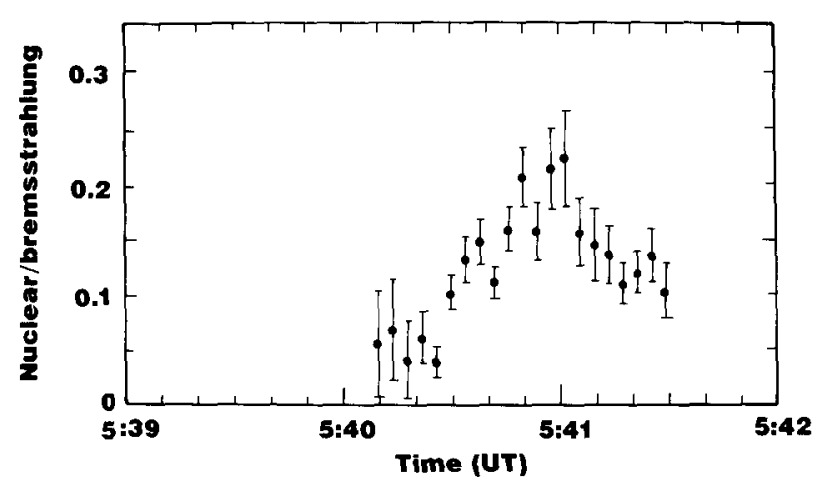

FiG. 6.-Temporal evolution of the ratio of counting rate in $4-7 \mathrm{MeV}$ band to counting rate in $0.4-0.7 \mathrm{MeV}$ band for the 1991 October 27 flare.

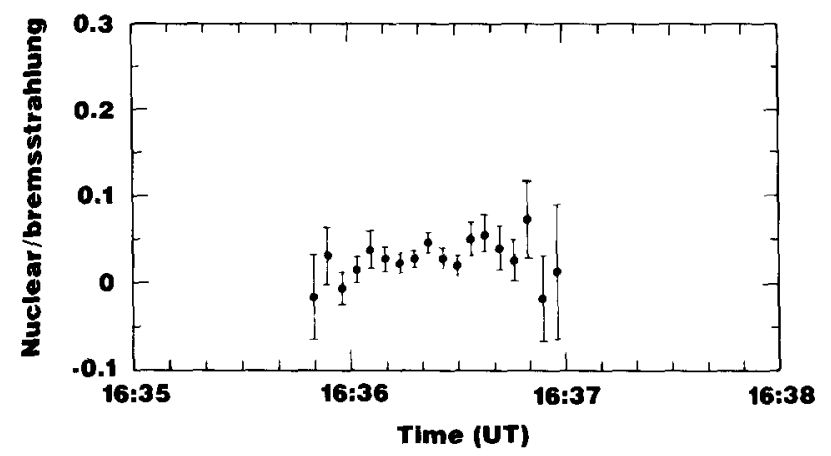

FIG. 7.-Temporal evolution of the ratio of counting rate in $4-7 \mathrm{MeV}$ band to counting rate in $0.4-0.7 \mathrm{MeV}$ band for the 1991 December 3 flare.

When the thick target interaction model with the stochastic acceleration spectrum given by a modified second-order Bessel function (Ramaty 1979) is assumed for the gamma-ray production, the values of the spectral parameter $\alpha T$ for the October 27 and November 15 flares are $>0.012$ and $0.010 \pm$ 0.002 , respectively. For the October 27 flare the lower limit of $\alpha T$ value is derived because the delayed $2.22 \mathrm{MeV}$ line could not fully observed (data coverage was partial, as mentioned above). Ramaty et al. (1993) summalized $\alpha T$ values derived for 20 flares observed so far. The $\alpha T$ values derived for these flares range from 0.01 to 0.02 . The present $\alpha T$ values lie within the range mentioned above.

The spectral profiles of 429 and $478 \mathrm{keV}$ lines from Be and Li deexcitation depends on the angular distribution of the interacting He nuclei (Murphy, Kozlovsky, \& Ramaty 1988; Murphy et al. 1990). For most gamma-ray flares, it seems difficult to detect the $\mathrm{Be}$ and $\mathrm{Li}$ deexcitation lines, because these lines are masked by a strong electron bremsstrahlung continuum during the maximum phase. The continuum-subtracted count spectrum for the disk-centered flare of November 15 is shown in Figure 8 . The calculated count spectra for three angular distributions (strong pitch-angle scattering, no pitch-angle scattering and isotropic distribution) are compared with the observed count spectra. In the case of strong pitch-angle scattering, the bulk of accelerated particles interact

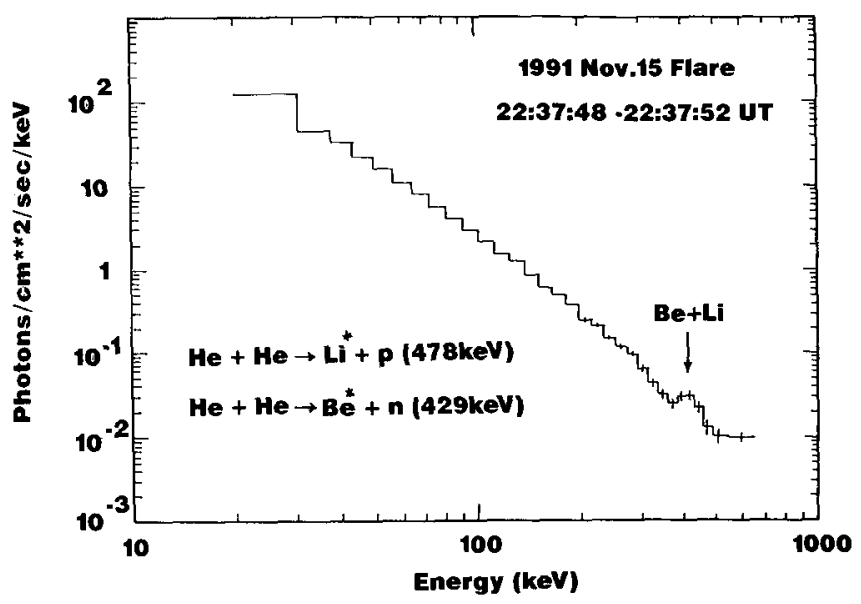

Fig. 8.-Be-Li line feature for the 1991 November 15 flare 
in the loss cone, leading to a distribution which is peaked in the downward direction. On the other hand, in the case of no pitch-angle scattering, the angular distribution peaks in a direction tangential to the photosphere. Figure 8 shows that the calculated spectra for strong pitch-angle scattering provides comparatively good fit to the observed count spectrum. But the observed count spectrum is possibly consistent with the spectrum derived from the isotropic distribution. We need high-resolution spectral observations of the $\mathrm{Be}$ and $\mathrm{Li}$ feature to discuss the angular distribution of interacting He nuclei in detail.

The Be and Li feature was observed from the 1981 April 27 flare with the SMM gamma-ray spectrometer (Forrest 1983). From the spectral analysis, the anisotropy of the accelerated He nuclei and the particle transport models were discussed (Murphy et al. 1990). The data were inconsistent with the spectrum derived for a downward beam, but it is difficult to distinguish the spectrum derived with strong pitch-angle scattering from that derived with no pitch-angle scattering. The data are also consistent with the spectrum derived for an isotropic distribution of interacting He nuclei.

The positron annihilation line at $511 \mathrm{keV}$ was detected during the decay phase of the November 15 flare. The time profile of $502-532 \mathrm{keV}$ band which contains the $511 \mathrm{keV}$ line and the underlying continuum exhibits trend of a little long decay compared with those of electron bremsstrahlung and prompt gamma-ray lines, as shown in Figure 9. From the detailed analysis of the time profile in the $502-532 \mathrm{keV}$ band, Kawabata et al. (1993) suggested the following scenarios of the $511 \mathrm{keV}$ line emission: (1) the main source of positrons is the deexcitation of ${ }^{16} \mathrm{O}^{* 6.052}$ by positron-electron pair emission, where an asterisk denotes an excited state, (2) $\beta^{+}$emission nuclei of ${ }^{31} \mathrm{~S},{ }^{29} \mathrm{P}$, ${ }^{27} \mathrm{Si},{ }^{26 \mathrm{~m}} \mathrm{Al},{ }^{25} \mathrm{Al},{ }^{23} \mathrm{Mg},{ }^{19} \mathrm{Ne}$, and ${ }^{21} \mathrm{Na}$ (mean lives of $4-25 \mathrm{~s}$ ) are also important sources of positrons in the decay phase, and (3) the half of the positrons annihilates in the photosphere of density $10^{16} \mathrm{~cm}^{-3}$ with a very short time, and the remainder annihilates in the dense coronal region of density of $10^{13} \mathrm{~cm}^{-3}$ (Hiei et al. 1992; Feldman 1992) with a lifetime of $30 \mathrm{~s}$. In the last solar maximum, the strong $511 \mathrm{keV}$ line emission was observed from two intense flares of 1980 June 21 and 1982

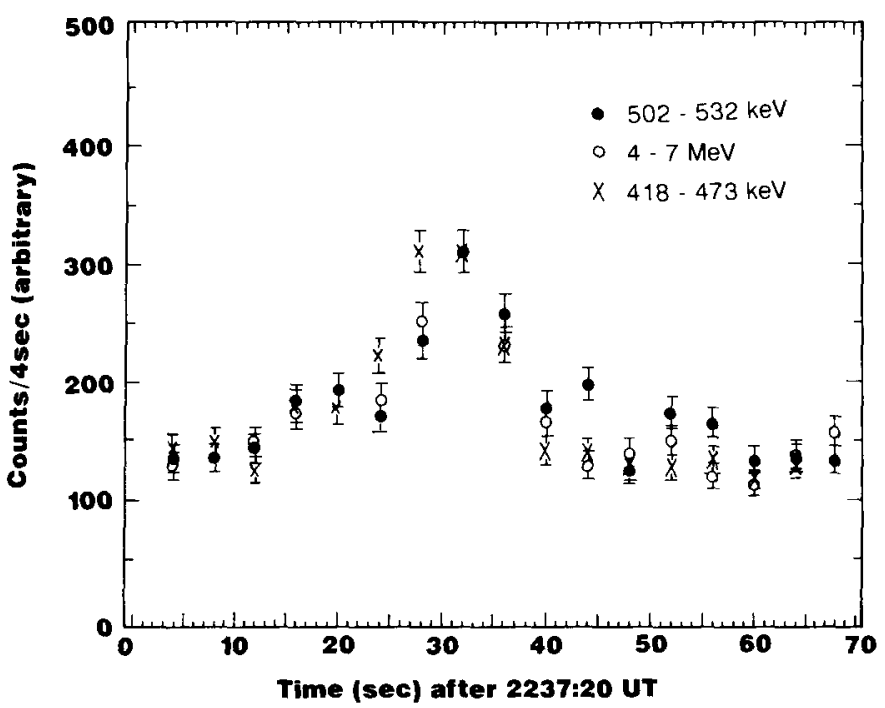

FIG. 9.-Time profile of the $502-532 \mathrm{keV}$ band for the 1991 November 15 flare. Time profiles of the $418-473 \mathrm{keV}$ (electron bremsstrahlung) and 4-7 MeV (prompt gamma-ray lines) are shown for comparison.

June 3 (Share et al. 1983). Murphy \& Ramaty (1984) showed that the time profile of the $511 \mathrm{keV}$ line for the flare of 1980 June 21 was determined primarily by the half-lives of $\beta^{+}$emitting nuclei. But the time profile for the flare of 1982 June 3 is more complicated and requires a more detailed study. Further observations of strong $511 \mathrm{keV}$ line emission are needed to provide arguments for the particle trapping in closed loops and for the positron annihilation region.

We appreciate the staff of the Institute of Space and Astronautical Science, the staff of the NASA Deep Space Network, and all of the Yohkoh team members for their support of the spacecraft operations. We thank Reuven Ramaty for helpful comments. This work is supported by Grant-in-Aid for Scientific Research of the Ministry of Education, Science and Culture (No. 03640285 and 04640311), and the Rikkyo University Grant for the Promotion of Research.
Chupp, E. L. 1987, Phys. Scripta, T18, 5

Feldman, U. 1992, preprint

Forrest, D. J. 1983, in Positron-Electron Pairs in Astrophysics, ed. M. L. Burns, A. K. Harding, \& R. Ramaty (New York: AIP), 3

Hiei, E., Nakagomi, Y., \& Takuma, H. 1992, PASJ, L77

Hua, X. M., \& Lingenfelter, R. E. 1987, Sol. Phys., 107, 351

Kozlovsky, B., \& Ramaty, R. 1974, ApJ, 191, L43

- 1977, Ap. Letters, 19, 19

Murphy, R. J., Hua, X. M., Kozlovsky, B., \& Ramaty, R. 1990, ApJ, 351 , 299

Murphy, R. J., Kozlovsky, B., \& Ramaty, R. 1988, ApJ, 331, 1029

Murphy, R. J., \& Ramaty, R. 1984, Adv. Space Res., 4, No. 7, 127

Ramaty, R. 1979, in Particle Acceleration Mechanisms in Astrophysics, ed. J. Arons, C. Max, \& C. Mckee (New York: AIP) 135

Ramaty, R., Mandzhavidze, N., Kozlovsky, B., \& Skibo, J. 1993, Adv. Space Res., 13, 275

\section{REFERENCES}

Rieger, E. 1989, Sol. Phys., 121, 323

Rieger, E., \& Marschhäuser, H. 1990, in Proc. Max '91 Workshop No. 3, Max '91/SMM Solar Flares, ed. R. M. Winglee \& A. L. Kiplinger (Boulder: Univ. of Colorado), 68

Share, G. H., Chupp, E. L., Forrest, D. J., \& Rieger, E. 1983, in PositronElectron Pairs in Astrophysics, ed. M. L. Burns, A. K. Harding, \& R. Ramaty (New York: AIP), 15

Yoshimori, M. 1989, Space Sci. Rev., 51, 85

Yoshimori, M., et al. 1991, Sol. Phys. 136, 69

Yoshimori, M., Takai, Y., Morimoto, K., Suga, K., \& Ohki, K. 1992a, PASJ, 44, L107

Yoshimori, M., et al. 1992b, PASJ, 44, L51

Yoshimori, M., \& Watanabe, H. 1985, J. Phys. Soc. Japan, 54, 2100 